\title{
GSM- R and CDMA Network System for the Railway Industry
}

\author{
T. Brandejský \& T. Vu Dinh* \\ Faculty of Transportation Sciences- Czech Technical University in Prague,Czech Republic \\ *Corresponding author: vudinhthem@yahoo.com
}

\begin{abstract}
Relates to the CDMA (Code Division Multiple Access) network with covering specifications and requirements which must be added in to the CDMA system. The Preliminary job is to analyze the demands and technologicaly development, mainly dealing mainly with the viability of the CDMA system in the railway industryrailway. Communication for rail transportation is vital to impulse stimulate the development of modern society. The CDMA system can bring the benefits from of voice and data services for to today's rail transportation.
\end{abstract}

KEY WORDS: CDMA network, railway, GSM-R

\section{INTRODUCTION OF MOBILE COMMUNICATION DEVELOPMENT}

The first cellular system were was deployed in the United state States in about in 1979, that called AMPS (Advanced Mobile Phone Service). and Iin the North-ern European countries, the system started operation in Sweden in 1981, and in Norway, Denmark and Finland with some manufacturers inventinged the NMT (Nordic Mobile Telephone) system. In 1985, TACS (Total Access Communication System) were was used in some European countries such as in the UK and Ireland. All these cellular systems used a frequency around $450 \mathrm{MHz}$ and /or around $900 \mathrm{MHz}$, and are called the first generation (1G) mobile phone systemss.

In 1990, the second generation (2G) mobile phone systems, such as GSM (Global System for Mobile communications) with standard IS-136, and CDMA with standard IS-95, were introduced in the United States and in Finland. Soon, the second generation digital mobile system was developed with an SMS service and reached a large number of subscribers number. The specification of a version of GSM adapted to the $1800 \mathrm{MHz}$ frequency band was added to the scope of the standardization group, with a frequency allocation of twice $75 \mathrm{MHz}$.

Shortly afterwards the third generation $(3 \mathrm{G})$ systems were researched (Lee and Miller, 1998). The first 3G network was launched by NTT DoCoMo in Japan in 2001 using the WCDMA (Wideband CDMA) technology (H. and A. Toskala, 2004). In tThe IMT-2000 standardization for $3 \mathrm{G}$ system supports a set of requirements such as data rates indoors reachesing $2 \mathrm{Mbit} / \mathrm{s}$ and $384 \mathrm{kbit} / \mathrm{s}$ outdoors. In 2002, the CDMA2000 1xEV-DO system was launched with high data rate services. Beside In the mean time, 2.5G systems such as CDMA $20001 \mathrm{x}$ and GPRS were extended from the existing $2 \mathrm{G}$ system (Yang, 2004). 
Characteristics of the next generation mobile communication are to support a larger number of subscribers and gain high data access speeds, as well as wider coverage. Up to now, with the rapid expansion of thein communication, many projects about on mobile communication have been implemented to satisfy the needs of telecommunications. In turn, mobile communication networks, from the first general $1 \mathrm{G}$ to $2 \mathrm{G}, 2.5 \mathrm{G}$, and now $3 \mathrm{G}$ and $4 \mathrm{G}$ networks using new technology, have been deployed all over the world, especially in the developed countries. By the end of 2007 there were 295 milion subscribers on $3 \mathrm{G}$ networks over the world. Revenue from $3 \mathrm{G}$ services makes uptotalled 120 billion dollars during 2007.

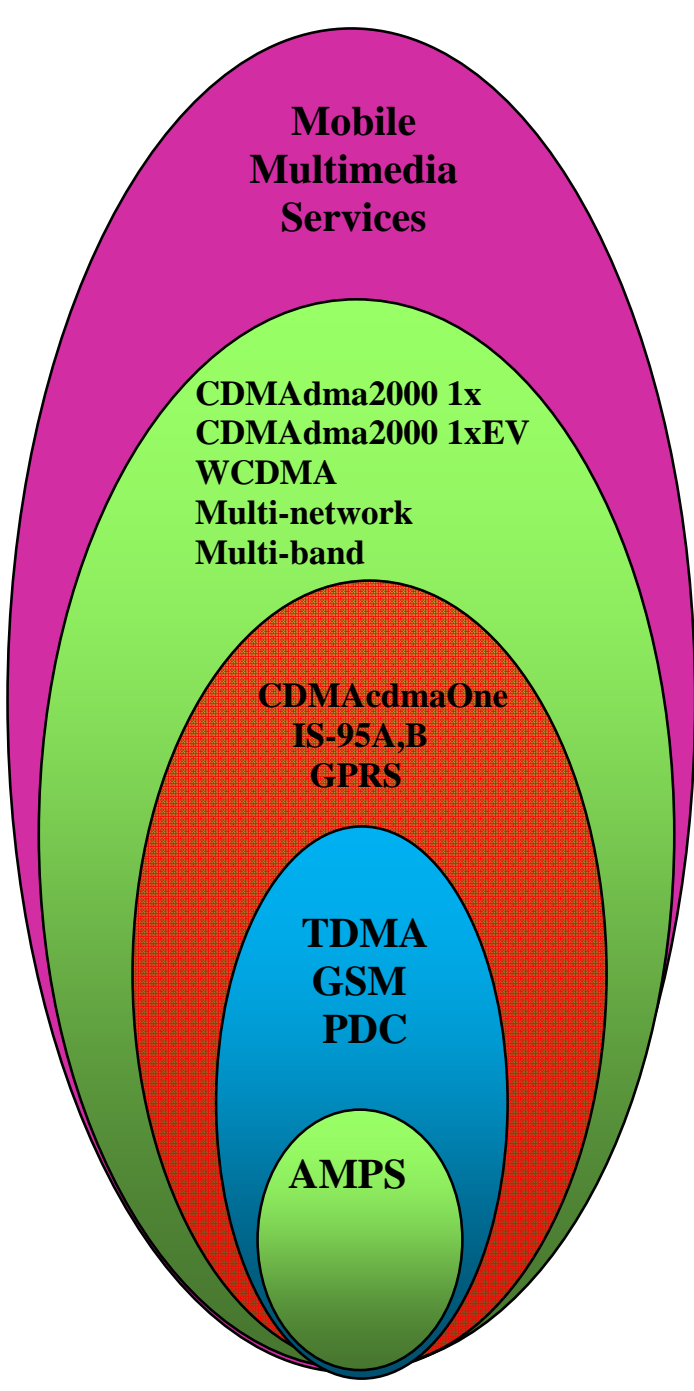

Figure 1: Development of mobile network generations time

3G(-2002)

4G(-2010)
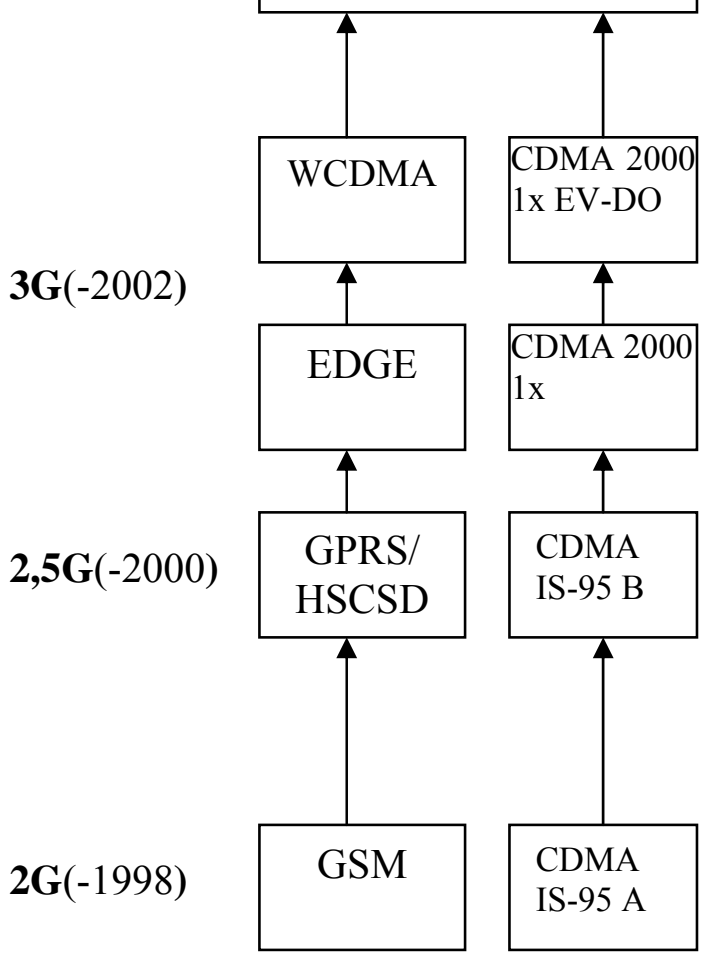

$1 G(-1979)$

\section{GSM-R FOR EUROPEAN RAILWAY NETWORK}

GSM-R (GSM-Railway) was the leader in MORANE trials and blazed the trail in commercial GSM-R deployments, rolling out a full-fledged infrastructure in 1998, deploying in 2000 in Sweden, and bringing the first high speed GSM-R application on line in Spain in 2003 (Nokia Siemens Networks, 2008). 


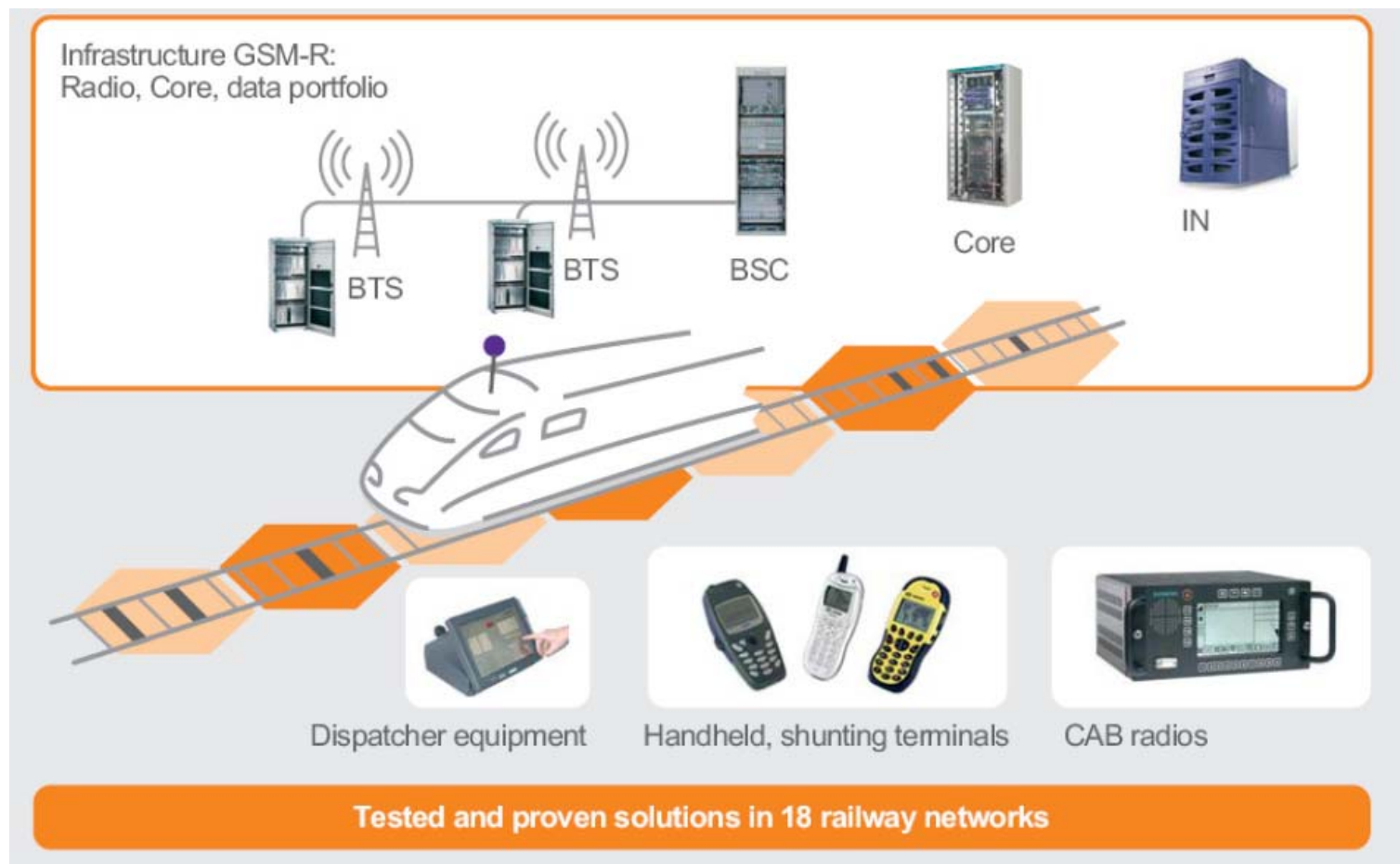

Figure 2: Infrastructure GSM-R (Nokia Siemens Network, 2008)

EIRENE (European Integrated Railway Radio Enhanced Network) specifications predetermine a radio system satisfying the mobile communications requirement of the European railway. These specifications consist of ground-train voice and data communication, together with the ground-base mobile communications needs of trackside workers, station and depot staff and railway administrative and managerial personal. The application of specification requirements will ensure interoperability for trains and staff crossing national and other borders between systems (Neele, 2007). The EIRENE specifications include:

EIRENE Functional Requirements Specification (E-FRS): this is a set of high-level functional requirements of the GSM-R railway radio system which facilitates international interoperability between national railways.

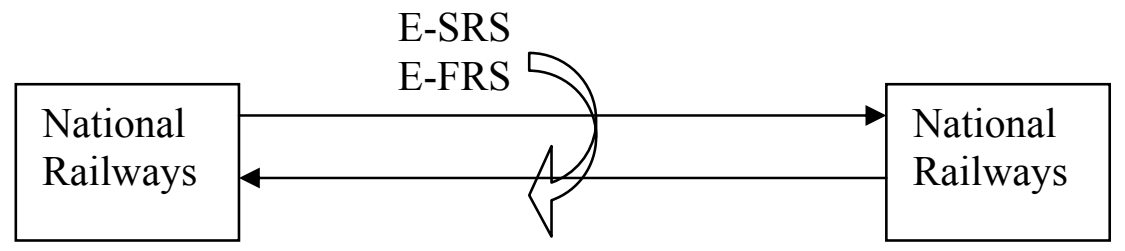

Figure 3: E-FRS and E-SRS facilitate and ensure internal railways

EIRENE system requirements specification (E-SRS) encompasses technical requirements and constraints in order to ensure international interoperability between national railways and support information as specification key to perform international interoperability.

MORANE specifications (Mobile radio for Railway Networks in Europe) consist of MORANE Sub System Requirements Specification (SSRS), MORANE Form Fit Functional Specification (FFFS), and MORANE Functional Interface Specifications (FIS). The SSRS certifies all the functions to perform on the GSM equipment in order to fulfill all 
requirements identified in the E-FRES and E-SRS. The FFFS indicates the technical system requirements defined in the higher level system specifications. The FIS defines the interfaces between network components.

The EIRENE and MORANE specifications are the minimum set of requirements necessary to ensure international interoperability of GSM-R network. The E-SRS can be included in a network service that provides an overview of the network services that must be supported by a mobile radio system and network planning that is to provide guidance on target performance levels for GSM coverage, hand-over and cell selection, call set-up time requirement and broadcast and group call areas. In addition, mobile equipment specification is taken by system specification as follows:

- Core specification: consist of basic services, facilities and features to ensure interoperability.

- Cab radio specification identifies the system requirements for the radio and the humanmachine interface and the functionality.

- General purpose radio requires the functions and physical properties taken from an EIRENE- compliant general purpose radio.

- Operational radio is basesd on the general purpose radio, but with the addition of functions to support railway operations.

- Numbering plan and call routing conclude numbering plan requirements, numbering plan constraints and structure of functional numbers and EIRENE numbering plan.

\section{CDMA NETWORK FOR RAILWAY}

In recent years, mobile communication networks have been rapidly researched and developed. Demands for multimedia services as voice, image and data are increasing more and more. The next generation mobile CDMA networks, which have been are being proposed now, offer high speed and a large capacity to satisfy multi-ple users. The system capacity of CDMA networks is a necessary problem for design engineering. The CDMA network shares a fixed resource, that is the frequency spectrum, to provide access channels for users.

A CDMA-R (CDMA-Railway) system is a mobile communication system for the railway industry which uses CDMA technology. Some specifications needed to satisfy for CDMA-R are the following: high safety and security, good cover quality, comfortable for the railway system, enable to link communication systems, roaming between other areas, integrated flexible high voice and data.

CDMA-R offers premium technical solutions, comprising of value added services, as well as train control, for example automatic ticketing cargo tracking and passenger information systems. The CDMA networks' lifecycle consists of design, implementation, and operation to long-term maintenance with using all the tools necessary for planning and managing networks and providing supporting services. Brief elements of CDMA-R include:

- Core network and associated equipments

- Interfaces to with railway equipments

- Roaming with other national and international networks

The CDMA-R also comprises of the same elements as same as the standards of the CDMA network:

- TE (terminal equipments): are user terminal equipments such as dispatcher equipment, handheld shunting terminals, and $\mathrm{CAB}$ radios. 
- BSC (base station controller): Functions of ilts function are is to controls a group of BTSs (Base Transceiver Station) that are connected. BSC has a function as a mobility management when a mobile moves from one BTS to the next BTS. and another important function of the BSC is transcoding. Transcoding converts the voice format used in the air interface and the voice format used in the PSTN.

- MSC (mobile switching center) is to switches connections between the MS (Mobile subscribers) and PSTN (public Public sServiceswitcheds telephone Telephone networkNetwork) or between the MS and another MSC.

- HLR (home location register): A database contains home subscriber information. Each subscriber, the HLR holds her the subscriber information, such as the international mobile subscriber identity (IMSI) and the selected long distance carrier.

- VLR (visitor location register): A database contains visiting subscriber information which is active on a current MSC.

- $\mathrm{AC}$ (authentication center): the AC authenticates user to provide service to that user.

- IWF (interworking function) that is to perform circuit switched data service. The IWF converts from one transmission format to another, and vice versa. Data flow originatinged from the MS is switched by the MSC to the IWF. The IWF converts the data stream into modem and transmits it over the PSTN.

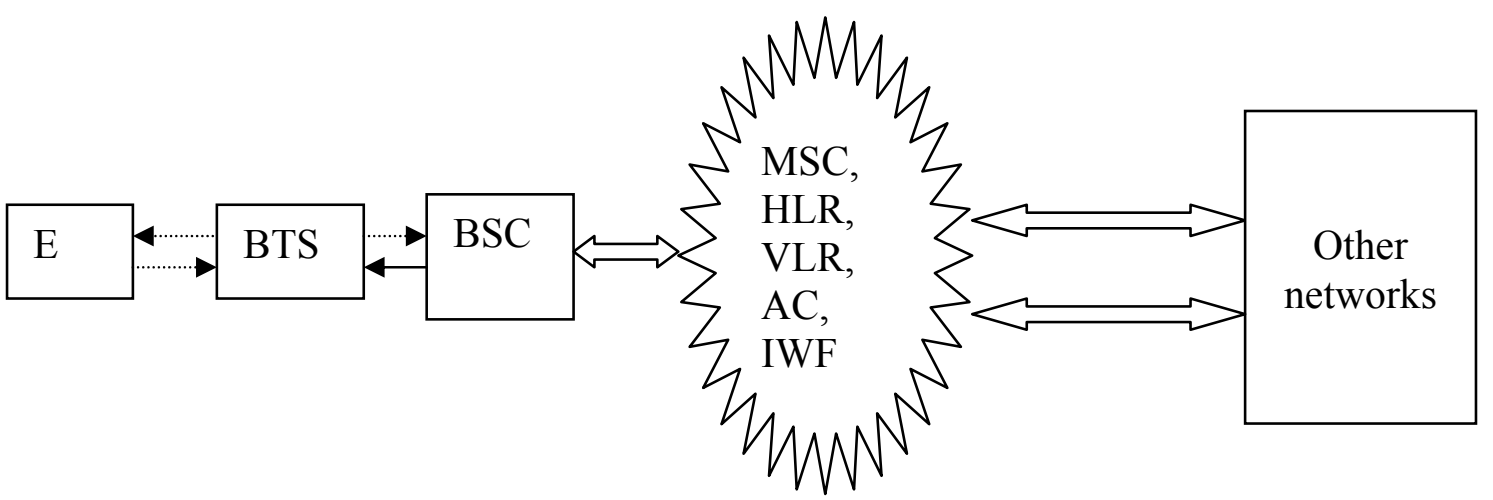

Figure 4: Simple structure of CDMA network

\section{ADVANTAGES AND DISADVANTAGES OF CDMA-R}

One of the main advantages of CDMA systems is their ability to use signals that arrive in the receivers with different time delays (Qualcomm, 2002). So the CDMA-R will resolve multipath phenomenon to improve link quality. The CDMA-R is based on code division multiple access technique, each user uses a common frequency with a different code that saves resources, which that make it a cost-effective, high quality wireless solution, easy and exact handoff. Fast and accurate power control to ensure that the base station receives all signals at the appropriate power. A CDMA rake receiver identifies the three strongest multi-path signals and combines them to achieve one very strong signal, so reducinge the power the transmitter must send, easy handoff.

Disadvantages of CDMA technology is channel pollution because due toof the same frequency. Otherwise, CDMA-R is not popular yet due to the expense of the technology and the starting of the GSM-G service.

TDMA (Time DMA) triples the capacity of FDMA (Frequency DMA), but CDMA capacity can be up to seven times that of TDMA. 
Table 1: Comparison of multiple access systems (Qualcomm, 2000)

\begin{tabular}{|l|c|c|c|c|c|c|}
\hline & \multicolumn{3}{|c|}{ Cellular band } & \multicolumn{3}{c|}{ PSC band } \\
\hline & CDMA & TDMA & FDMA & CDMA & TDMA & FDMA \\
\hline Channel bandwidth & $1.25 \mathrm{MHz}$ & $30 \mathrm{KHz}$ & $30 \mathrm{KHz}$ & $1.25 \mathrm{MHz}$ & $30 \mathrm{KHz}$ & $200 \mathrm{KHz}$ \\
\hline Users/sector/channel & $22 / 14$ & 3 & 1 & $22 / 14$ & 3 & 8 \\
\hline Standard & IS-95A & IS-54 & EIA/TIA-553 & ANSI-J-STD008 & IS-136 & GSM \\
\hline
\end{tabular}

\section{CONCLUSION}

This paper mentions some rasespects of railway telecommunication to take the discussion to CDMA-R. This will begin the process and lead to the design of a private railway communication network that has applied CDMA technology perfectly. The role of telecommunications in railways is very important. It ensures the safe working of trains such as the signaling or train control function and also supports other train operations, without difficulty, a high safety response and security.

\section{ACKNOLEDGEMENT}

The work has been supported by Czech Ministry of Education No. MSM 6840770043. This support is very gratefully acknowledged.

\section{REFERENCES}

HEINE, G.: GSM Networks, Artech House, 1998.

HOLMA, H., TOSKALA, A.: WCDMA for UMTS, John Wiley \& Sons, 2004.

LEE, J. S., MILLER, L. E.: CDMA Systems Engineering Handbook, Artech House, 1998.

MOULY, M., PAŇTET, M.: The GSM System for Mobile Communications, Palaiseau France, 1992.

NEELE, A. F.: GSM-R system, 2007.

TORRIERI, D.: Principles of Spread- Spectrum Communication Systems, Spring, 2005.

YANG, S. C.: 3G CDMA 2000 Wireless System Engineering, Artech House, 2004.

PRASAT, R., MUNOZ, L.: WLANs and WPANs towards 4G Wireless, Artech House, 2003.

Nokia Siemens Networks, GSM-R tomorrow's communication for today's rail transportation, 2008.

Qualcomm, cdmaONE and CDMA2000, Qualcomm incorporated, 2002.

Qualcomm, Introduction to CDMA, Qualcomm Learning center, 2000. 